\title{
Tetramer orbital ordering and lattice chirality in $\mathrm{MnTi}_{2} \mathrm{O}_{4}$
}

\author{
A. Rahaman, ${ }^{1}$ M. Chakraborty ${ }^{2}$ T. Paramanik,${ }^{1,3}$ R. K. Maurya, ${ }^{4}$ S. Mahana,${ }^{5}$ R. Bindu,${ }^{4}$ \\ D. Topwal, ${ }^{6,7}$ P. Mahadevan, ${ }^{8}$ and D. Choudhury ${ }^{1, *}$ \\ ${ }^{1}$ Department of Physics, Indian Institute of Technology Kharagpur, Kharagpur-721302, India \\ ${ }^{2}$ Centre for Theoretical Studies, Indian Institute of Technology Kharagpur, Kharagpur-721302, India \\ ${ }^{3}$ Department of Physics, School of Sciences, National Institute of Technology Andhra Pradesh, Tadepalligudem- 534102, India \\ ${ }^{4}$ School of Basic Sciences, Indian Institute of Technology Mandi-Kamand, Himachal Pradesh-175005, India \\ ${ }^{5}$ Rajdhani College, Bhubaneswar-751003, India \\ ${ }^{6}$ Institute of Physics, Sachivalaya Marg, Bhubaneswar-751005, India \\ ${ }^{7}$ Homi Bhabha National Institute, Training School Complex, Anushakti Nagar, Mumbai 400085, India \\ ${ }^{8}$ S. N. Bose National Center for Basic Sciences, Block JD, Salt Lake, Kolkata-700098, India
}

(Received 1 April 2019; revised manuscript received 14 August 2019; published 30 September 2019)

\begin{abstract}
The presence of orbital degree of freedom in strongly correlated systems leads to unusual orderings. In this paper we use a combination of density-functional theory calculations and various experimental investigations to reveal a unique ground state for a $\mathrm{Ti}^{3+}$ containing spinel oxide, $\mathrm{MnTi}_{2} \mathrm{O}_{4}$, which hosts an exotic combination of a rare tetramer orbital (associated with $\mathrm{Ti}^{3+} 3 d^{1}$ electron) ordering along equivalent $\langle 111\rangle$ directions involving all three $t_{2 \mathrm{~g}}$ orbitals, a ferrimagnetic $\mathrm{Mn}$-Ti, and a ferromagnetic Ti-lattice spin ordering. A combination of spin-orbital superexchange and Jahn-Teller-effect related strain-energy optimization provides a microscopic understanding for the stabilization of the unique ground state, which is found to be also electrically polar. The tetramer orbital ordering induces Ti-Ti bond length modulations and the short and long Ti-Ti bonds form helices around the crystallographic $c$ axis with a particular winding direction, causing the ground state structure to become spatial chiral.
\end{abstract}

DOI: 10.1103/PhysRevB.100.115162

\section{INTRODUCTION}

Transition-metal (TM) oxides with orbital degrees of freedom constitute one of the most fascinating fields of research in condensed-matter physics and hosts copious physical phenomena, which include high-temperature superconductivity, colossal magnetoresistance, and multiferroicity [1-3]. In transition-metal oxides with strong electron-electron correlations, electrons are primarily localized on the atoms. Exotic physics ensue when such localized electrons also possess orbital degrees of freedom, i.e., electrons can choose to occupy between a set of equivalent and energy-degenerate atomic orbitals. Octahedrally coordinated $\mathrm{Mn}^{3+}$ ions in $\mathrm{LaMnO}_{3}$ with $3 d^{4}\left(t_{2 \mathrm{~g}}^{3}-e_{\mathrm{g}}^{1}\right)$ configuration constitutes a representative example, where a single electron has a choice to occupy any of the two degenerate $e_{\mathrm{g}}$ orbitals. Often at a lower temperature, the electron chooses one from the two $e_{\mathrm{g}}$ orbitals, which breaks the local charge symmetry and is accompanied by differential oxygen-ion displacements, referred to as JahnTeller (JT) distortion. In a solid, such choices on different atoms are interdependent, which results in cooperative JT distortions associated with a spontaneous orbital-ordering (OO) transition, wherein localized occupied orbitals on various ions form a regular pattern $[2,4-6]$. Similar to the $e_{\mathrm{g}}$ OO systems, transition-metal oxides constituting ions possessing $t_{2 \mathrm{~g}}$-level orbital degrees of freedom, such as in $\mathrm{YTiO}_{3}\left(\right.$ one $\mathrm{Ti}^{3+} d$

*debraj@phy.iitkgp.ac.in electron in a subspace of three degenerate $t_{2 \mathrm{~g}}$ orbitals) [7,8], $\mathrm{MnV}_{2} \mathrm{O}_{4}$ (two $\mathrm{V}^{3+} d$ electrons among three degenerate $t_{2 \mathrm{~g}}$ orbitals) [9-11], also exhibit cooperative JT distortions and various $\mathrm{OO}$ ground states.

Mostly, in these TM systems, either a ferro-OO state (similar occupied orbital at all ionic sites) or an antiferro-OO state (with alternate ions occupied by similar orbitals), or a combination of the two along different directions is realized. The presence of higher-order $\mathrm{OO}$ has very few examples, such as $\mathrm{CuIr}_{2} \mathrm{~S}_{4}$ [12,13], $\mathrm{MgTi}_{2} \mathrm{O}_{4}$ [13,14], and $\mathrm{Fe}_{3} \mathrm{O}_{4}$ [15-17], and unlike the simpler examples discussed earlier, the forces driving the $\mathrm{OO}$ largely remain a puzzle.

In this paper, we report a unique tetramer $\mathrm{OO}$ state in spinel oxide $\mathrm{MnTi}_{2} \mathrm{O}_{4}$ (which contains $\mathrm{Mn}^{2+} \mathrm{O}_{4}$ tetrahedral and $\mathrm{Ti}^{3+} \mathrm{O}_{6}$ octahedral units; the $\mathrm{Ti}$ sites alone forming a pyrochlore lattice). As this is unusual, we use a combination of theory and experiments to explore the driving mechanism for the orbital ordering. The ground-state lattice and magnetic structure of $\mathrm{MnTi}_{2} \mathrm{O}_{4}$, however, remains contentious [18-20]. We elucidate that the ferrimagnetic tetragonal $P 4_{1}$ structure is the ground state of $\mathrm{MnTi}_{2} \mathrm{O}_{4}$ and show that this structure hosts a unique combination of tetramer OO, lattice-chirality and spontaneous electric polarization. As the levels in one spin channel on $\mathrm{Mn}$ are filled, superexchange interactions between $\mathrm{Mn}$ and Ti sites result in an antiferromagnetic Mn-Ti coupling. This in turn leads to a ferromagnetic coupling between the spins on $\mathrm{Ti}^{\mathrm{Ti}} \mathrm{Ti}^{3+}\left(3 d^{1}\right)$ ions in octahedral coordination are JT active and so while one can envisage few patterns of orbital ordering consistent with a ferromagnetic Ti lattice, in this 
system we find that the strain energy costs are lowest when a tetramer ordering is favored. Thus, besides the identification of an unusual orbital-ordering in $\mathrm{MnTi}_{2} \mathrm{O}_{4}$, we elucidate the microscopic mechanisms that drive it.

\section{METHODOLOGY}

$A b$ initio density-functional theory (DFT) calculations were performed using the all-electron full-potential augmented linearized plane-wave method and taking the augmented plane-wave basis as implemented in the WIEN2 $\mathrm{K}$ code [21]. In order to elucidate the ground state of $\mathrm{MnTi}_{2} \mathrm{O}_{4}$, relative energies between various spinel structures were investigated and spin-polarized calculations with different spin configurations were performed. Volume as well as internal geometries were optimized for all the investigated structures in the presence of on-site electron-electron correlation $(U)$ using the GGA-PBE exchange correlation functional [22]. Spin-orbit coupling was incorporated with GGA $+U$ calculations by the second variational code along with scalar relativistic corrections [23]; however, its effect was found to be negligible. Muffin-tin radii of 1.98, 1.80, and 1.50 a.u. for $\mathrm{Mn}, \mathrm{Ti}$, and $\mathrm{O}$, respectively, and a $k$-point mesh of $9 \times 9 \times 6$ were considered for all the calculations. $R k_{\max }, G_{\max }$, and $l_{\max }$ were set to 7.0, 14.0 $\mathrm{Bohr}^{-1}$, and 12, respectively. Throughout the paper, the $\mathrm{Ti}$ orbitals are defined in the local $\mathrm{TiO}_{6}$ coordinate system. The ferroelectric polarization calculations were performed using the Berry-phase method [24,25] with the Vienna $A b$ initio Simulation Package (VASP) [26]. For the experimental investigations, polycrystalline samples of $\mathrm{MnTi}_{2} \mathrm{O}_{4}$ were synthesized using a solid state reaction route from a mixture of $\mathrm{MnO}, \mathrm{TiO}_{2}$, and metallic $\mathrm{Ti}$ powders [19]. The mixture was ground well and sintered in the form of pellets at $900{ }^{\circ} \mathrm{C}$ under vacuum in a sealed quartz tube. The phase formation of the sample, which contained $\sim 5 \%$ of $\mathrm{Ti}_{2} \mathrm{O}_{3}$ as impurity phase (as reported earlier [18]), was established using the temperature-dependent $\mathrm{x}$-ray diffraction (XRD) technique and cell parameters were extracted from Rietveld refinement of XRD data using the FULLPROF package [27]. X-ray absorption near-edge structure (XANES) and extended $\mathrm{x}$-ray absorption fine-structure (EXAFS) measurements were carried out to investigate the electronic and local-crystallographic structures at various temperatures at the P-65 beamline of the PETRA III synchrotron source, DESY, Hamburg, Germany. The preprocessing and fitting of EXAFS data were carried out over the $k$ range of $3-12 \AA^{-1}$ using ATHENA and ARTEMIS softwares [28].

\section{THEORETICAL RESULTS}

\section{A. Ground-state structure}

Cubic $F d \overline{3} m$ as well as tetragonal $I 4_{1} /$ amd (which is a simple elongation of cubic $F d \overline{3} m$ along the $c$ axis) structures have been proposed to be the ground state of $\mathrm{MnTi}_{2} \mathrm{O}_{4}$ [18-20]. We, however, find that the ferrimagnetic $P 4_{1}$ tetragonal structure possesses the lowest energy $(56 \mathrm{meV} /$ f.u. smaller than the closest cubic $F d \overline{3} m$ structure; the $U$ values used for these calculations and energies of other competing structures are detailed in Tables I and II of the Supplemental Material (SM) file [29]). Between the cubic $F d \overline{3} m$ structure
TABLE I. Structural parameters of the ground state of $\mathrm{MnTi}_{2} \mathrm{O}_{4}$-Space group: Tetragonal $P 4_{1}, \quad a=6.154 \AA$, $c=8.699 \AA$.

\begin{tabular}{lcccc}
\hline \hline Atom & $x$ & $y$ & $z$ & Site \\
\hline $\mathrm{Mn}$ & 0.242 & 0.753 & 0.250 & $4 a$ \\
$\mathrm{Ti}(1)$ & 0.742 & 0.496 & 0.371 & $4 a$ \\
$\mathrm{Ti}(2)$ & 0.742 & 0.996 & 0.378 & $4 a$ \\
$\mathrm{O}(1)$ & 0.513 & 0.748 & 0.384 & $4 a$ \\
$\mathrm{O}(2)$ & 0.971 & 0.741 & 0.392 & $4 a$ \\
$\mathrm{O}(3)$ & 0.521 & 0.256 & 0.365 & $4 a$ \\
$\mathrm{O}(4)$ & 0.979 & 0.247 & 0.358 & $4 a$ \\
\hline \hline
\end{tabular}

and tetragonal $I 4_{1} /$ amd structure of $\mathrm{MnTi}_{2} \mathrm{O}_{4}$, the $F d \overline{3} m$ structure is found to have a lower energy, which is consistent with the earlier report [20]. Further, small changes in the atomic positions from the corresponding high-symmetric tetragonal I41/amd and $I 41 / a$ structures, in the ferrimagnetic GGA $+U$ calculations, converges into the $P 4_{1}$ ground state. $\mathrm{MgTi}_{2} \mathrm{O}_{4}$ spinel oxide, which also contains $\mathrm{Ti}^{3+}$ ions, has a chiral tetragonal $P 4_{1} 2_{1} 2$ ground-state structure containing Tiion spin singlets [30], and all reported results are reproduced well using our GGA $+U$ calculations. As a further check of the ground state of $\mathrm{MnTi}_{2} \mathrm{O}_{4}$, we have performed GGA $+U$ calculations without any symmetry restriction on the $P 4_{1} 2_{1} 2$ ground-state structure of $\mathrm{MgTi}_{2} \mathrm{O}_{4}$ after replacement of the $\mathrm{Mg}^{2+}$ ions with $\mathrm{Mn}^{2+}$ ions and have obtained the symmetryreduced $P 4_{1}$ structure. As shown in Table I, the obtained ground-state $P 4_{1}$ structure of $\mathrm{MnTi}_{2} \mathrm{O}_{4}$ consists of two inequivalent $\mathrm{Ti}$ sites, and thus has a lower symmetry than the $I 4_{1} /$ amd and $P 4_{1} 2{ }_{1} 2$ tetragonal structure, which has only one Ti site. Interestingly, the $P 4_{1}$ structure is noncentrosymmetric, with both $\mathrm{Ti}$ and $\mathrm{Mn}$ ions displaced from the center of the $\mathrm{TiO}_{6}$ octahedral and $\mathrm{MnO}_{4}$ tetrahedral cages, respectively. The calculated ferroelectric polarization value of the $P 4_{1}$ structure of $\mathrm{MnTi}_{2} \mathrm{O}_{4}$ is $0.5 \mu \mathrm{Ccm}^{-2}$.

Calculations performed using different $U$ values, like $(2,2)$, $(3,2)$, and $(3,3) \mathrm{eV}$ for $(\mathrm{Mn}, \mathrm{Ti})$ ions ( $U$ values taken in accordance with earlier calculations on related systems [11,14,31]), give similar density of states (DOS) and same OO pattern but differ in their band-gap values. Figure 1 shows the spinresolved DOS in the ground-state $P 4_{1}$ structure of $\mathrm{MnTi}_{2} \mathrm{O}_{4}$, evaluated using a GGA $+U$ calculation $[U=(3,2) \mathrm{eV}$ for (Mn,Ti) ions]. The total DOS near the Fermi level is dominated by Ti DOS and its splitting gives rise to an insulating band gap of $0.26 \mathrm{eV}$. As seen in Fig. 1(a), the up-spin channel is nearly completely occupied for the Mn atoms, validating its $2+\left(3 d^{5}\right)$ high-spin state. The up-spin channel for Ti atoms is nearly empty, signifying the ferrimagnetic configuration where Mn (with only up-spin levels occupied) and Ti spins are aligned antiferromagnetically and the Ti spins arranged ferromagnetically. The near-orthogonal superexchange interactions between $\mathrm{Mn}-\mathrm{Ti}$ ions (average $\mathrm{Mn}^{2+}-\mathrm{O}^{2-}-\mathrm{Ti}^{3+}$ bond angle is $\sim 123^{\circ}$ ) and $\mathrm{Ti}-\mathrm{Ti}$ ions (average $\mathrm{Ti}^{3+}-\mathrm{O}^{2-}-\mathrm{Ti}^{3+}$ bond angle is $\sim 95.9^{\circ}$ ) in $\mathrm{MnTi}_{2} \mathrm{O}_{4}$, following GoodenoughKanamori-Anderson rules [32], is also in accordance with the obtained ferrimagnetic coupling between $\mathrm{Mn}^{2+}$ (with only one hopping-spin channel available) and $\mathrm{Ti}^{3+}$ ions and a 

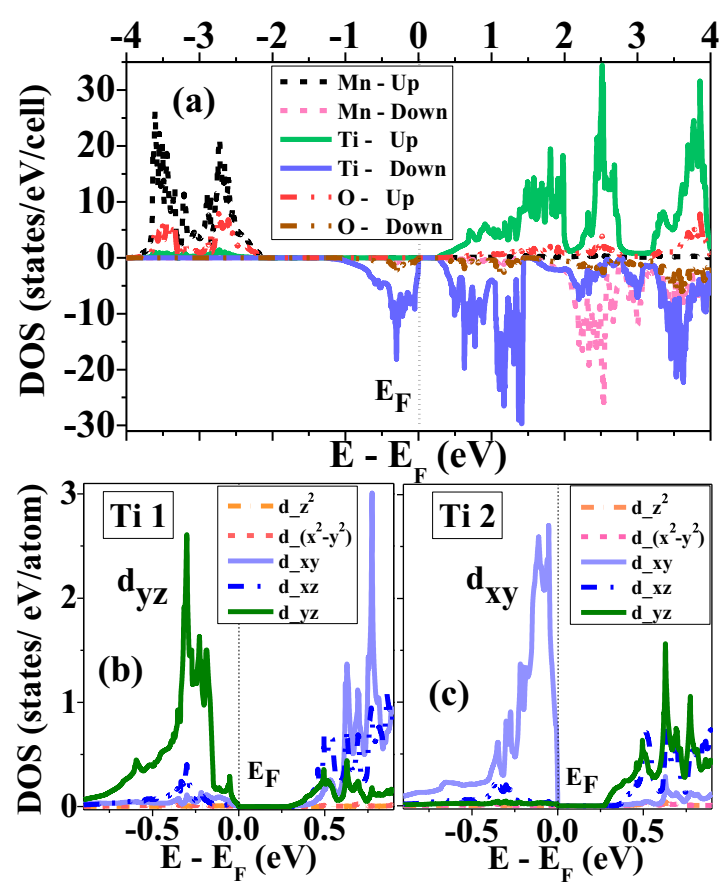

FIG. 1. Density-of-states (DOS) of the $P 4_{1}$ structure of $\mathrm{MnTi}_{2} \mathrm{O}_{4}$ : (a) total DOS for all atoms (Mn, Ti, and O), (b) for Ti1 $d$ levels, and (c) for Ti2 $d$ levels in the down-spin channel.

ferromagnetic $\mathrm{Ti}^{3+}$ lattice. Further, Figs. 1(b) and 1(c) show the orbitally resolved partial DOS for the two inequivalent $\mathrm{Ti}$ atoms, Ti1 and Ti2. Clearly, the $t_{2 \mathrm{~g}}$-level degeneracy of the $\mathrm{TiO}_{6}$ octahedra of the cubic state is broken in the tetragonal phase through JT distortions and the single $\mathrm{Ti}^{3+}-3 d^{1}$ electron dominantly occupies a single $t_{2 \mathrm{~g}}-d$ level, which varies between the Ti atoms. The occupied $t_{2 \mathrm{~g}}$ orbital is one among the $a_{1 \mathrm{~g}}\left(d_{x z}\right.$ or $\left.d_{y z}\right)$ orbitals for the Til atoms and the $e_{g}^{\prime}$ $\left(d_{x y}\right)$ orbital for the Ti2 atoms. The $d_{x z}, d_{y z}$, and $d_{x y}$ orbital occupancies are associated with the shortest Ti-O bond being along the $y, x$, and $z(c)$ directions, respectively. Instead of two inequivalent $\mathrm{Ti}$ atoms, the presence of a single kind of $\mathrm{Ti}$ atom would have either led to a spin-singlet pairing (indicated by diagonal blue lines) among the Ti spins [30,33,34], as indicated in Figs. 2(a) or 2(b), or resulted in an unsustainable piling of strain along certain crystallographic directions from accompanying JT effects, as indicated in Fig. 2(c). The presence of two distinct Ti sites, as shown in Fig. 2(d), ensures a ferromagnetic coupling between the $\mathrm{Ti}^{3+}$ ions, and also results in an effective distribution of the shortest Ti-O bonds amongst different crystallographic directions, thereby leading to a reduction of the cooperative JT-effect-related strain energy, as shown in Figs. 2(d) and 2(f).

\section{B. Orbital Ordering}

The obtained charge-density plots of the occupied Ti $d$ orbitals (shown in Fig. 2 of SM [29]) illustrates the emergence of $\mathrm{OO}$ in the ground state involving all three $t_{2 \mathrm{~g}}$ orbitals $\left(d_{x z}\right.$, $d_{y z}$, and $d_{x y}$ ). In the subsequent $a b$ planes, the OO pattern remains the same, only the character of the participating orbitals vary between either the $d_{x z}-d_{x y}$ or the $d_{y z}-d_{x y}$ pairs. Interestingly, a unique tetramer-OO, i.e., a $d_{y z}-d_{x z}-d_{x y}-d_{x y}$ ordering, is

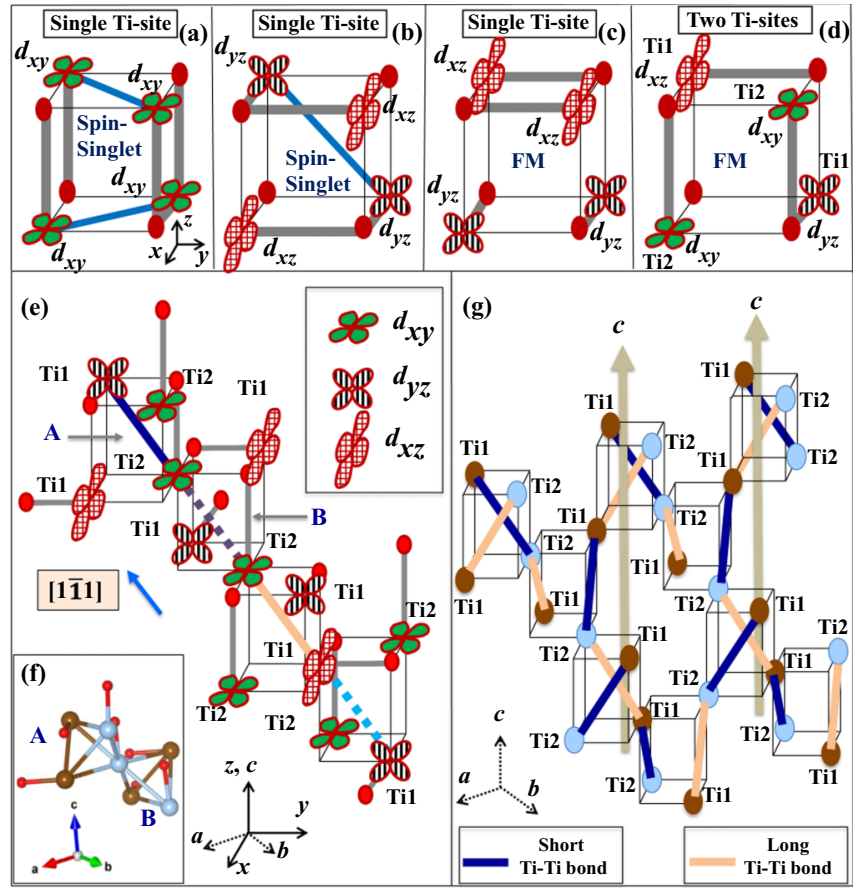

FIG. 2. Illustration showing various possible distributions of Tisite orbital occupancies among the Ti tetrahedra resulting from JahnTeller distortions for [(a)-(c)] a single kind of Ti atom and (d) two kinds of $\mathrm{Ti}$ atoms in the spinel structure. For each $\mathrm{Ti}$ atom, the direction of the shortest Ti-O bond is illustrated by thick gray lines. Small red balls are the $\mathrm{O}$ atoms. Direct overlap of similar occupied orbitals in (a) and (b) would result in the formation of spin-singlet dimers, which are highlighted by thick blue diagonal lines. (e) Illustration showing the Ti $d$-level $-d_{y z}-d_{x z}-d_{x y}-d_{x y}$-tetramer orbital ordering along the [1111] direction of the crystal unit cell of $\mathrm{MnTi}_{2} \mathrm{O}_{4}$. The obtained Ti $d$ orbital ordering is accompanied with Ti-Ti bond-length modulations (the solid dark-blue, dashed blue, solid light-brown, and dashed sky-blue colored Ti-Ti bond lengths are $3.010 \AA$, $3.13 \AA$, $3.14 \AA$, and $3.03 \AA$, respectively.) (f) The spatial distribution of the shortest Ti-O bond distances in the corner-shared Ti tetrahedral network of $\mathrm{MnTi}_{2} \mathrm{O}_{4}$. (g) Short and long Ti-Ti bonds, when joined, form helices around the crystallographic $c$ axis of $\mathrm{MnTi}_{2} \mathrm{O}_{4}$.

observed for the Ti chains running along the equivalent $\langle 111\rangle$ directions [29]. The tetrahedrons comprising two distinct Ti sites, as shown in Fig. 2(d), form interconnected chains and a unique tetramer atomic ordering (Ti1-Ti1-Ti2-Ti2) along the equivalent $\langle 111\rangle$ directions [as shown in Fig. 2(e)]. Similar considerations of the distribution of shortest Ti-O bonds amongst three orthogonal directions and a reduction of the JTeffect-related strain energy, are effective in giving rise to the two distinct Ti sites in Fig. 2(d), also becomes effective for the interconnected Ti chains along equivalent $\langle 111\rangle$ directions, resulting in a unique tetramer-OO $\left(d_{y z}-d_{x z}-d_{x y}-d_{x y}\right)$ state, as shown in Fig. 2(e). Modulations in Ti-Ti bond distances are necessarily associated with such cooperative JT distortions. We identify four Ti-Ti bond distances in the tetramer $\mathrm{OO}$ state [shown in Fig. 2(e)]. Interestingly, among these four Ti -Ti bonds, the short and the long Ti-Ti bonds, when joined together, form helices with a particular winding direction along the crystallographic $c$ direction, causing the structure to become lattice chiral [illustrated in Fig. 2(g)]. We also 

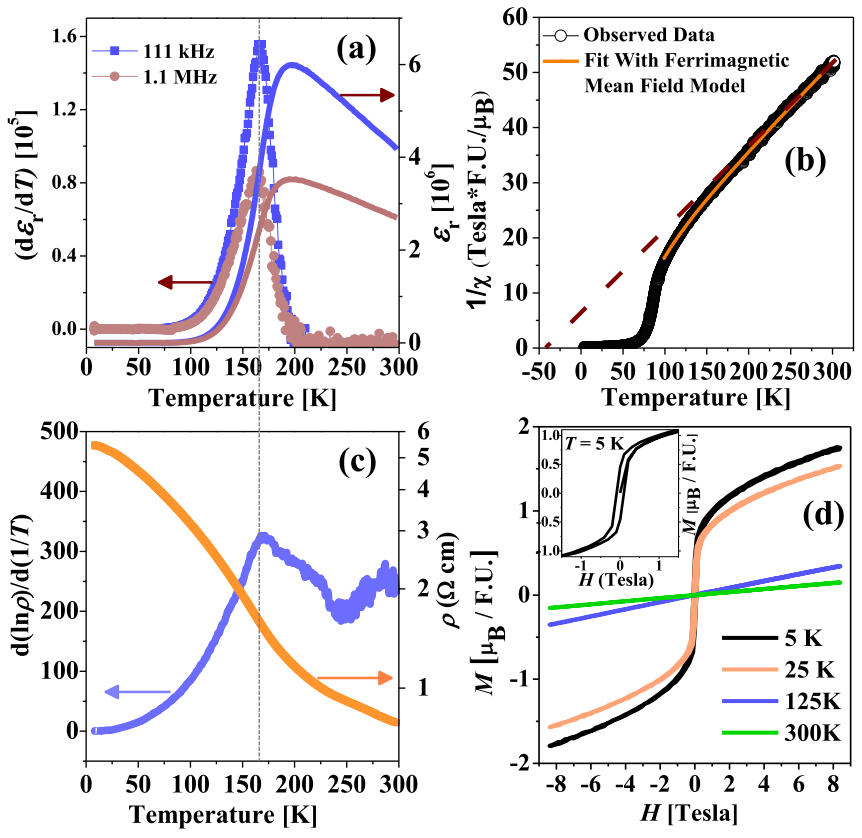

FIG. 3. Temperature ( $T$ ) dependencies of (a) dielectric constant $\left(\varepsilon_{\mathrm{r}}\right)$ and $\frac{d \varepsilon_{\mathrm{r}}}{d T}$, (b) inverse susceptibility $\left(\frac{1}{\chi}\right)$ measured with a magnetic field $(H)$ of 0.1 Tesla (the solid line is a fit using a mean-field ferrimagnetic model and the dashed line is a guide to the eye), and (c) resistivity $(\rho)$ and $\frac{d \ln \rho}{d(1 / T)}$ of $\mathrm{MnTi}_{2} \mathrm{O}_{4}$. (d) Isothermal $M-H$ curves measured at $5,25,125$, and $300 \mathrm{~K}$ with the inset showing an expanded view of the hysteresis loop at $5 \mathrm{~K}$.

find that a tetragonal $P 4_{3}$ structure is degenerate in energy to the $P 4_{1}$ structure. These two structures vary in the sense of the lattice chirality (one is left-handed chiral and the other is right-handed chiral) but are similar otherwise.

\section{EXPERIMENTAL RESULTS}

\section{A. Electronic and Structural Properties}

To corroborate the theoretical findings of a structural transition, we next discuss the results of experimental investigations on $\mathrm{MnTi}_{2} \mathrm{O}_{4}$. The temperature dependence of the dielectric constant of $\mathrm{MnTi}_{2} \mathrm{O}_{4}$ is plotted in Fig. 3(a). The dielectric constant rises sharply from low temperature and its derivative exhibits a clear peak at $\sim 164 \mathrm{~K}$. Importantly, this peak position does not disperse with varying electric-field frequencies, which is indicative of a ferroelectric transition [35]. A clear transition can also be easily discerned at $\sim 164 \mathrm{~K}$ in the plots of the temperature dependencies of the resistivity and scaled effective activation energy (in units of K), as shown in Fig. 3(c). Temperature-dependent XRD studies were carried out to investigate the presence of a structural transition around this temperature range. Differential XRD peak broadenings were indeed observed for $\mathrm{MnTi}_{2} \mathrm{O}_{4}$ with a lowering of temperature. For some representative XRD peaks (the characteristic right shoulder arises from the $\mathrm{Cu} k_{\beta}$ satellite), such as (311), (400), (511), and (440), the peaks were found to be broader at lower temperatures $(15$ and $100 \mathrm{~K}$ ) compared to the $300 \mathrm{~K}$ spectrum (the former comparison shown in Fig. 4(a) and the latter three comparisons are shown in Fig. 2 of [29]). We note that this behavior is opposite to what is expected from a usual
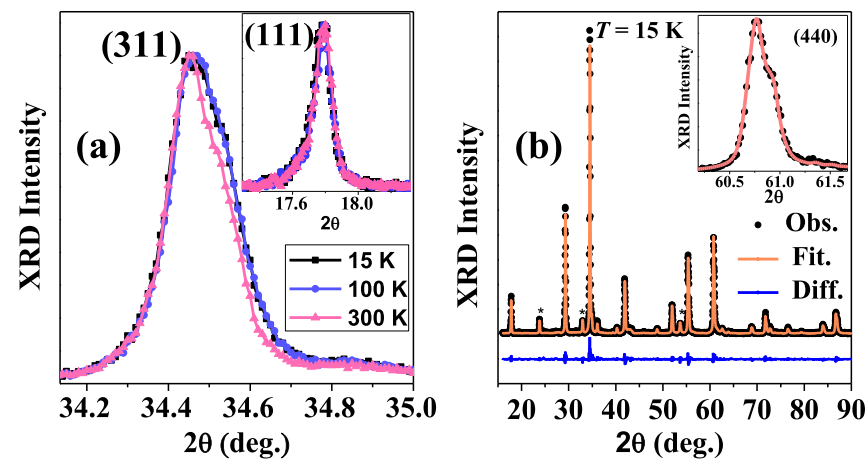

FIG. 4. (a) Broadening of (311) x-ray diffraction (XRD) peaks measured at $T=15$ and $100 \mathrm{~K}$ with respect to that measured at $T=300 \mathrm{~K}$. Inset shows that similar $T$-dependent broadening is not observed for (111) XRD peak. (b) Fitting of XRD pattern at $T=$ $15 \mathrm{~K}$ with a $P 4_{1}$ tetragonal structure. The refinement also includes $\sim 5 \% \mathrm{Ti}_{2} \mathrm{O}_{3}$ impurity phase, whose peaks are marked with asterisks.

thermal-broadening effect. The (111) XRD peak of $\mathrm{MnTi}_{2} \mathrm{O}_{4}$, as shown in the inset to Fig. 4(a), however, exhibits no additional broadening with lowering of temperature. The observed differential XRD peak broadenings are consistent with [18], where through careful synchrotron-based $\mathrm{x}$-ray diffraction studies on $\mathrm{MnTi}_{2} \mathrm{O}_{4}$ the authors were able to unearth subtle evidence of a structural transition from high-temperature cubic to low-temperature tetragonal structure. Rietveld refinements of the XRD pattern of $\mathrm{MnTi}_{2} \mathrm{O}_{4}$ at $15 \mathrm{~K}$ were performed considering various structures, such as the cubic $F d \overline{3} m$, orthorhombic $F d d d$, tetragonal $I 4_{1} /$ amd, and tetragonal $P 4_{1}$. We find that the best refinement for the low-temperature $(T=15 \mathrm{~K})$ XRD pattern [shown in Fig. 4(b)] is obtained using the tetragonal noncentrosymmetric enantiomorphic $P 4_{1}$ structure $\left(\chi^{2}\right.$ of 1.50$)$. To investigate the low- $T$ structural distortions in further details, we have carried out temperaturedependent Ti- $K$-edge XANES and EXAFS spectroscopic studies on $\mathrm{MnTi}_{2} \mathrm{O}_{4}$. First, we discuss the spectral shape of the Ti- $K$-edge XANES spectrum which is a bulk-sensitive probe for the valence state of the $\mathrm{Ti}$ ions. The comparisons of the Ti- $K$-edge spectral shape of $\mathrm{MnTi}_{2} \mathrm{O}_{4}$ with the standard reference spectra, as shown in Fig. 5, establish the presence of $\mathrm{Ti}$ ions in the $(3+)$ valence state in nearly stoichiometric $\mathrm{MnTi}_{2} \mathrm{O}_{4}$, consistent with the estimated valence state. We next discuss the corresponding EXAFS oscillations, an excellent probe of local structural distortions, which is complementary to the bulk-sensitive XRD technique. In the following, we focus on analyses of EXAFS oscillations at the Ti- $K$-edge recorded on either side of the estimated structural transition temperature of $\sim 164 \mathrm{~K}$, i.e., 32 and $200 \mathrm{~K}$. The observed EXAFS oscillations at $32 \mathrm{~K}$, as shown in Fig. 6(a), were fitted better (both qualitatively in terms of matching spectral shape and quantitatively in terms of a lower fitting-related $R$ factor) when using the tetragonal structure than the cubic structure. Also, at $200 \mathrm{~K}$, the EXAFS oscillations were fitted better with the cubic phase than the tetragonal structure [as shown in Fig. 6(b)]. Thus, both bulk-sensitive XRD and local-probe EXAFS investigations compliment each other well and suggest a low-temperature $P 4_{1}$ tetragonal ground-state structure for $\mathrm{MnTi}_{2} \mathrm{O}_{4}$, in accordance with our theoretical results. 


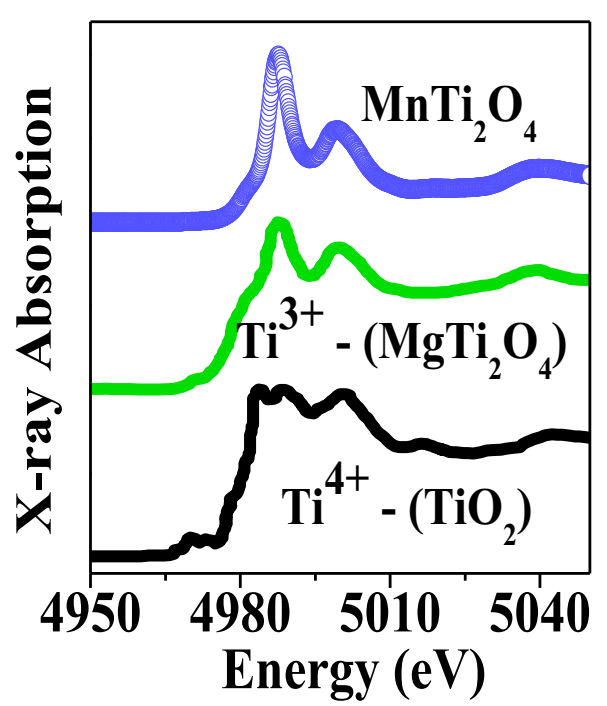

FIG. 5. Comparison of Ti- $K$-edge XANES spectra of $\mathrm{MnTi}_{2} \mathrm{O}_{4}$ with $\mathrm{MgTi}_{2} \mathrm{O}_{4}$ (which contains $\mathrm{Ti}^{3+}$ ions) [36] and $\mathrm{TiO}_{2}$ (which contains $\mathrm{Ti}^{4+}$ ions) [37].

\section{B. Magnetic Properties}

The magnetization $(M)$ of $\mathrm{MnTi}_{2} \mathrm{O}_{4}$ rises sharply by lowering the temperature $(T)$ around $50 \mathrm{~K}$. A magnetic transition around $45 \mathrm{~K}$ is estimated from the peak position in the derivative of $M-T$ data (the small impurity phase of $\mathrm{Ti}_{2} \mathrm{O}_{3}$, which has a spin-singlet transition at $\sim 450 \mathrm{~K}$ [38], does not affect the magnetic data in the investigated temperature range), which is consistent with corresponding electron spin resonance data [19]. The low-field magnetization value of $\mathrm{MnTi}_{2} \mathrm{O}_{4}$, as observed here, is larger when compared to that reported in Ref. [18], possibly due to the presence of additional magnetic disorder in the latter sample (both $\mathrm{Ti}_{2} \mathrm{O}_{3}$ and $\mathrm{MnTiO}_{3}$ impurity phases have been reported along with the main $\mathrm{MnTi}_{2} \mathrm{O}_{4}$ phase in Ref. [18]). The small low-field magnetization value recovered and increased through doping of $\mathrm{V}$ in place of $\mathrm{Ti}$ ions [18]. To explain the above observations, the magnetic ground state of $\mathrm{MnTi}_{2} \mathrm{O}_{4}$ has been reported to be paramagnetic in Ref. [18]. To investigate the nature of the magnetic ordering, we focus on the $T$ dependence of the $\frac{1}{x}$ data, which exhibits a clear low- $T$ hyperbolic shape and a negative $T$ intercept, as shown in Fig. 3(b), which, however, is
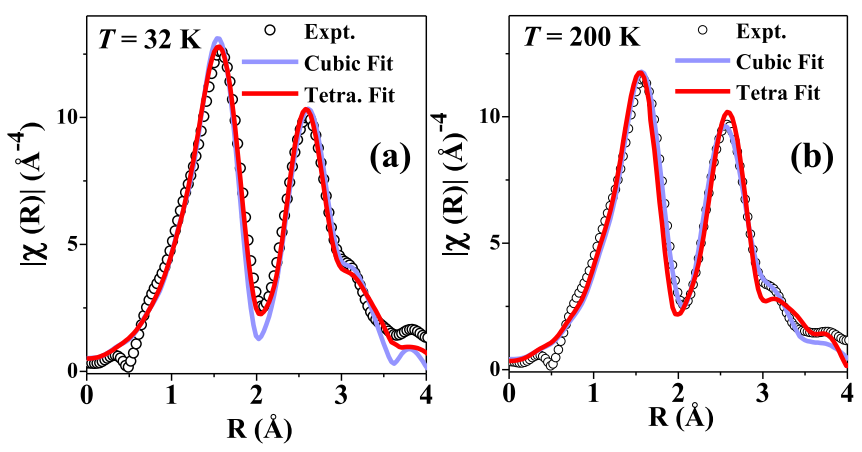

FIG. 6. Comparative fittings of Ti- $K$-edge EXAFS oscillations at (a) $T=32 \mathrm{~K}$ and (b) $200 \mathrm{~K}$ with tetragonal and cubic structures. in accordance with a ferrimagnetic ordering. The magnetic $\frac{1}{x}$ data, in the complete high-temperature paramagnetic region, can be fitted well using a mean-field ferrimagnetic model, which considers two magnetic sublattices [39]. In $\mathrm{MnTi}_{2} \mathrm{O}_{4}$, these two magnetic sublattices are clearly identified with the $\mathrm{Mn}$ and Ti lattices. Further, the negative temperature intercept of the $\frac{1}{x}$ data (which can be extracted reliably from a simple linear extraplotaion of the high-temperature data and is, thus, independent of the fitting protocol), i.e., $-43 \mathrm{~K}$, clearly establishes an antiferromagnetic interaction between the two magnetic sublattices [39] and a ferrimagnetic ground state. Clear observations of $M$ vs magnetic field $(H)$ loops below $45 \mathrm{~K}$ [shown in the inset of Fig. 3(d)] further support the presence of a ferrimagnetic transition around $45 \mathrm{~K}$ for $\mathrm{MnTi}_{2} \mathrm{O}_{4}$, which is in excellent agreement with our theoretical results. The magnetization, measured at $5 \mathrm{~K}$, continues to increase at high fields, possibly due to the polycrystalline nature of our sample due to contributions from magnetic domains with magnetization directions perpendicular to the applied field direction. We note that the collinear ferrimagnetic ordering of $\mathrm{MnTi}_{2} \mathrm{O}_{4}$, as proposed here, is also consistent with the rise in magnetization value with increasing $\mathrm{V}$ content observed in the solid solution of $\mathrm{MnTi}_{2} \mathrm{O}_{4}$ and $\mathrm{MnV}_{2} \mathrm{O}_{4}$ [18] (since the latter has a larger magnetization value due to spin-canting of $\mathrm{V}$ moments away from the Mn moment [9]).

\section{ROLE OF Mn}

It is instructive to compare the obtained results on $\mathrm{MnTi}_{2} \mathrm{O}_{4}$ vis-à-vis those of $\mathrm{MgTi}_{2} \mathrm{O}_{4} \cdot \mathrm{MgTi}_{2} \mathrm{O}_{4}$ undergoes a concomitant cubic to tetragonal $\left(P 4_{1} 2_{1} 2\right.$ ground-state structure containing one Ti site) structural transition and a spinsinglet magnetic transition [30]. The differences in the structural, electronic, and magnetic configurations between these two systems are driven primarily by the $\mathrm{Mn}-\mathrm{Ti}$ antiferromagnetic superexchange interactions in $\mathrm{MnTi}_{2} \mathrm{O}_{4}$, which favors a completely contrasting ferromagnetic Ti lattice. Such interactions are absent in $\mathrm{MgTi}_{2} \mathrm{O}_{4}$ since the $A$-site $\mathrm{Mg}^{2+}$ ion has a closed electronic shell and is, thus, diamagnetic. In addition to Mn-Ti superexchange interactions, considerations of the reduction of Jahn-Teller-effect-related strain energy, as discussed earlier, plays a major role in selecting the unique tetramer orbital-ordered ground state for $\mathrm{MnTi}_{2} \mathrm{O}_{4}$, as shown in Fig. 2(e), which involve all three $t_{2 \mathrm{~g}}$ orbitals.

\section{CONCLUSION}

Using $a b$ initio DFT calculations and a combination of several experimental techniques we have elucidated a unique $P 4_{1}$ tetragonal ground state of $\mathrm{MnTi}_{2} \mathrm{O}_{4}$, which hosts a unique combination of tetramer $\mathrm{OO}$, ferroelectricity, and lattice chirality. The obtained Ti-site spin and orbital configurations are in good agreement with results from model calculations involving spin-orbital-superexchange interactions of threefold orbitally degenerate $S=\frac{1}{2}$ ions on a general pyrochlore lattice [33]. We find that a combination of SE interactions among $\mathrm{Mn}$ and $\mathrm{Ti}$ spins and the minimization of cooperative JT-effect-related strain energy become instrumental for the stabilization of the unique ground state in $\mathrm{MnTi}_{2} \mathrm{O}_{4}$. 


\section{ACKNOWLEDGMENTS}

D.C. would like to gratefully acknowledge SRIC-IIT Kharagpur (ISIRD grant), SERB, DST (funding under project file No. ECR/2016/000019), BRNS, and DAE (funding through sanction No. 37(3)/20/23/2016-BRNS) for financial support. T.P. would like to acknowledge SERB for providing

[1] B. Keimer, Nat. Mater. 5, 933 (2006).

[2] Y. Tokura and N. Nagaosa, Science 288, 462 (2000).

[3] S.-W. Cheong, Nat. Mater. 6, 927 (2007).

[4] Y. Murakami, J. P. Hill, D. Gibbs, M. Blume, I. Koyama, M. Tanaka, H. Kawata, T. Arima, Y. Tokura, K. Hirota, and Y. Endoh, Phys. Rev. Lett. 81, 582 (1998).

[5] Y. Murakami, H. Kawada, H. Kawata, M. Tanaka, T. Arima, Y. Moritomo, and Y. Tokura, Phys. Rev. Lett. 80, 1932 (1998).

[6] P. Mahadevan, K. Terakura, and D. D. Sarma, Phys. Rev. Lett. 87, 066404 (2001).

[7] J. Varignon, M. N. Grisolia, D. Preziosi, P. Ghosez, and M. Bibes, Phys. Rev. B 96, 235106 (2017).

[8] M. Mochizuki and M. Imada, New J. Phys. 6, 154 (2004).

[9] V. O. Garlea, R. Jin, D. Mandrus, B. Roessli, Q. Huang, M. Miller, A. J. Schultz, and S. E. Nagler, Phys. Rev. Lett. 100, 066404 (2008).

[10] T. Suzuki, M. Katsumura, K. Taniguchi, T. Arima, and T. Katsufuji, Phys. Rev. Lett. 98, 127203 (2007).

[11] S. Sarkar, T. Maitra, R. Valenti, and T. Saha-Dasgupta, Phys. Rev. Lett. 102, 216405 (2009).

[12] P. G. Radaelli, Y. Horibe, M. J. Gutmann, H. Ishibashi, C. H. Chen, R. M. Ibberson, Y. Koyama, Y.-S. Hor, V. Kiryukhin, and S. W. Cheong, Nature (London) 416, 155 (2002).

[13] D. I. Khomskii and T. Mizokawa, Phys. Rev. Lett. 94, 156402 (2005).

[14] S. Leoni, A. N. Yaresko, N. Perkins, H. Rosner, and L. Craco, Phys. Rev. B 78, 125105 (2008).

[15] H.-T. Jeng, G. Y. Guo, and D. J. Huang, Phys. Rev. Lett. 93, 156403 (2004).

[16] M. S. Senn, J. P. Wright, and J. P. Attfield, Nature (London) 481, 173 (2012).

[17] J. P. Wright, J. P. Attfield, and P. G. Radaelli, Phys. Rev. Lett. 87, 266401 (2001).

[18] T. Sonehara, K. Kato, K. Osaka, M. Takata, and T. Katsufuji, Phys. Rev. B 74, 104424 (2006).

[19] Y. Huang, Z. Yang, and Y. Zhang, J. Magn. Magn. Mater. 324, 2075 (2012).

[20] T. Zhang, Z. L. Lv, Y. Cheng, X. R. Chen, and G. F. Ji, Comput. Mater. Sci. 84, 156 (2014).

[21] P. Blaha, K. Schwarz, G. K. H. Madsen, K. Kvasnicka, and J. Luitz, WIEN2k (Karlheinz Schwarz, Techinische Universitat Wien, Austria, 2001). support through a fellowship (file No. PDF/2016/002580). S.M. and D.T. would like to gratefully acknowledge financial support by DST provided within the framework of the India@DESY collaboration. A.R. and D.C. would like to acknowledge Swastika Chatterjee, Poonam Kumari, Partha Pratim Jana, Arghya Taraphder, and Dibyendu Dey for support and various fruitful discussions.
[22] J. P. Perdew, K. Burke, and M. Ernzerhof, Phys. Rev. Lett. 77, 3865 (1996).

[23] D. D. Koelling and B. N. Harmon, J. Phys. C: Solid State Phys. 10, 3107 (1977).

[24] R. D. King-Smith, and D. Vanderbilt, Phys. Rev. B 47, 1651(R) (1993).

[25] R. Resta, Rev. Mod. Phys. 66, 899 (1994).

[26] G. Kresse and J. Hafner, Phys. Rev. B 47, 558 (1993); 48, 13115 (1993); 49, 14251 (1994).

[27] T. Roisnel and J. Rodŕnguez-Carvajal, Materials Science Forum: Proceedings of the Seventh European Powder Diffraction Conference (EPDIC 7), Vol. 378-381, edited by R. Delhez and E. J. Mittenmeijer (Trans Tech Publications, Barcelona, Spain, 2000), pp. 118-123.

[28] B. Ravel and M. Newville, J. Synchrotron Radiat. 12, 537 (2005).

[29] See Supplemental Material at http://link.aps.org/supplemental/ 10.1103/PhysRevB.100.115162 for energy comparison tables and charge-density plot obtained from GGA + U calculations, structural parameters obtained from Rietveld refinement and representative XRD peaks at few selected temperatures.

[30] M. Schmidt, W. Ratcliff, II, P. G. Radaelli, K. Refson, N. M. Harrison, and S. W. Cheong, Phys. Rev. Lett. 92, 056402 (2004).

[31] D. Dey, T. Maitra, and A. Taraphder, Phys. Rev. B 93, 195133 (2016).

[32] D. I. Khomskii, Transition Metal Compounds (Cambridge University Press, Cambridge, UK, 2014).

[33] S. Di Matteo, G. Jackeli, C. Lacroix, and N. B. Perkins, Phys. Rev. Lett. 93, 077208 (2004).

[34] M. Isobe, and Y. Ueda, J. Phys. Soc. Jpn. 71, 1848 (2002).

[35] Measurement of a ferroelectriclike electric-polarization vs electric-field loop measurement was, however, not successful due to the lossy-semiconducting nature of the sample.

[36] O. Durmeyer, J. P. Kappleri, E. Beaurepairet, and J. M. Heintz, J. Phys.: Condens. Matter 2, 6127 (1990).

[37] G. A. Waychunas, Am. Mineral. 72, 89 (1987).

[38] C. F. Chang, T. C. Koethe, Z. Hu, J. Weinen, S. Agrestini, L. Zhao, J. Gegner, H. Ott, G. Panaccione, H. Wu, M. W. Haverkort, H. Roth, A. C. Komarek, F. Offi, G. Monaco, Y.-F. Liao, K.-D. Tsuei, H.-J. Lin, C. T. Chen, A. Tanaka, and L. H. Tjeng, Phys. Rev. X 8, 021004 (2018).

[39] J. S. Smart, Am. J. Phys. 23, 356 (1955). 